\title{
Suprachoroidal Injection of Triamcinolone- Review of a Novel Treatment for Macular Edema Caused by Noninfectious Uveitis
}

\author{
Kenneth W Price, ${ }^{1}$ Thomas A Albini, ${ }^{2}$ and Steven Yeh ${ }^{1}$
}

1. Department of Ophthalmology, Emory University School of Medicine, Atlanta, GA, USA; 2. Bascom Palmer Eye Institute, University of Miami Miller school of Medicine, Miami, FL, USA

DOI: https://doi.org/10.17925/USOR.2020.13.2.76

$\mathrm{M}$ acular edema is the most frequent cause of visual deterioration in noninfectious uveitis. The treatment of noninfectious uveitis with associated macular edema commonly includes systemic or locally administered corticosteroids, with long-term use limited by significant side effects. The need for a treatment with an improved safety profile has driven the development of a novel ophthalmic therapy: a proprietary triamcinolone acetonide suspension (CLS-TA) administered in the suprachoroidal space (XIPERE ${ }^{\mathrm{TM}}$; Clearside Biomedical, Alpharetta, GA, USA). Suprachoroidal delivery of corticosteroids allows higher steroid concentration in the posterior segment and decreases the risk of other adverse ocular events. Recent results from the PEACHTREE trial (ClinicalTrials.gov Identifier: NCT02595398), a phase III trial with two suprachoroidal injections of CLS-TA at 0 and 12 weeks with follow up lasting 24 weeks, showed the significant improvement in visual acuity and reduction in central subfield thickness, all without increasing the risk of elevated intraocular pressure or accelerated cataract progression.

\section{Keywords}

Suprachoroidal injection, macular edema, uveitis, steroid

Disclosure: Kenneth W Price, Thomas A Albini, and Steven Yeh have no financial or non-financial relationships or activities to declare in relation to this article.

Review Process: Double-blind peer review.

Compliance with Ethics: This study involves a review of the literature and did not involve any studies with human or animal subjects performed by any of the authors.

Authorship: The named authors meet the International Committee of Medical Journal Editors (ICMJE) criteria for authorship of this manuscript, take responsibility for the integrity of the work as a whole, and have given final approval for the version to be published. Access: This article is freely accessible at touchOPHTHALMOLOGY.com (C) Touch Medical Media 2021 Received: July 29, 2020

Accepted: November 2, 2020

Published Online: January 27, 2021

Citation: US Ophthalmic Review. 2020;13(2):76-9

Corresponding Author: Steven Yeh, 1365 Clifton Road Building B T401A, Atlanta, GA, 30322, USA.

E: steven.yeh@emory.edu

Support: This project was supported by the National Eye Institute/ National Institutes of Health core grant P30-EY06360 (Department of Ophthalmology, Emory University School of Medicine) and R01 EY029594 (Steven Yeh). The content is solely the responsibility of the authors and does not necessarily represent the official views of the National Institutes of Health or the views or policies of the Department of Health and Human Services, nor does mention of trade names, commercial products, or organizations imply endorsement by the US Government. This research was also supported an unrestricted departmental grant from Research to Prevent Blindness, Inc. to the Emory Eye Center, Emory University School of Medicine.
Uveitis consists of a wide array of inflammatory disorders of the uveal tract and surrounding structures, which may lead to irreversible ocular damage and lasting visual impairment if not appropriately treated in a timely manner. As the fifth most common cause of vision loss in the developed world, uveitis accounts for 3-10\% of legal blindness in the USA and Europe. ${ }^{1-4}$ The etiologies of uveitis may be infectious or noninfectious in origin. Noninfectious uveitis may be associated with systemic autoimmune diseases, or may be limited to the eye and can be classified as anterior, intermediate, posterior, or panuveitis according to the primary site of inflammation. ${ }^{5}$

Macular edema, a multifactorial structural complication of uveitis, involving the breakdown of the blood-retinal barrier, is the leading cause of vision loss associated with uveitis. ${ }^{1.6}$ The disease burden of macular edema is significant, and is observed in nearly one-third of all patients with uveitis, including $60 \%$ of patients with intermediate and panuveitis, $40 \%$ of individuals with posterior uveitis, and an estimated $20 \%$ of individuals with anterior uveitis. ${ }^{7}$ Therapeutic options for macular edema may include either systemic or locally administered immunomodulatory agents. Locally administered therapies primarily utilize corticosteroids and may be administered via topical eye drops, intraocular or periocular injections, or corticosteroid implant technology. ${ }^{8.9}$ The use of anti-vascular endothelial growth factor (VEGF) agents has been limited given its modest efficacy and increased frequency of injections compared with local corticosteroids. ${ }^{10}$

Corticosteroid delivery approaches may act via diffusion from periocular administration, redistribution via the vascular system after systemic absorption, or direct administration into the vitreous to target the disease process. Given the side effects of local therapies, including cataract and glaucoma, as well as the inefficient localization of medication when administered systemically, additional local treatment options with an improved safety spectrum are needed to help control macular edema associated with noninfectious uveitis. ${ }^{11-17}$

\section{The suprachoroidal space-anatomy and favorable drug kinetics}

The suprachoroidal space is a physiologic space between the sclera and choroid that traverses the circumference of the posterior segment of the eye. It is essential in maintaining intraocular pressure (IOP) via uveoscleral outflow and is an expandable space that is collapsed in physiologic conditions. ${ }^{18}$ Given its close proximity to the choroid, retinal pigment epithelium, and retina, the suprachoroidal space is an attractive target for pharmacotherapy. ${ }^{19}$ 
Table 1: Clinical trials utilizing suprachoroidal drug delivery

\begin{tabular}{|c|c|c|c|c|c|}
\hline Study name & Study type & $\begin{array}{l}\text { Disease } \\
\text { indication }\end{array}$ & $\mathrm{N}$ & Major efficacy outcome & Safety considerations \\
\hline $\begin{array}{l}\text { Safety Study of Suprachoroidal Triamcinolone } \\
\text { Acetonide via Microneedle to Treat Uveitis;, } \\
\text { ClinicalTrials.gov Identifier: NCT01789320 }\end{array}$ & Phase I/II & NIU & 9 & $\begin{array}{l}\text { Safe and tolerable; all patients } \\
\text { with improvement in visual acuity }\end{array}$ & $\begin{array}{l}\text { No systemic AEs related to study drug; } \\
\text { most common ocular AE was pain }\end{array}$ \\
\hline $\begin{array}{l}\text { Suprachoroidal Injection of Triamcinolone Acetonide } \\
\text { in Subjects with Macular Edema Following } \\
\text { Non-Infectious Uveitis (DOGWOOD):28 } \\
\text { ClinicalTrials.gov Identifier: NCT02255032 }\end{array}$ & Phase II & $\begin{array}{l}\text { ME due } \\
\text { to NIU }\end{array}$ & 22 & $\begin{array}{l}69 \% \text { of patients had decreased } \\
\text { central subfield thickness } \\
\text { of }>20 \% \text {, improved vision in } 65 \%\end{array}$ & No steroid-related increase in IOP \\
\hline $\begin{array}{l}\text { Suprachoroidal Injection of CLS-TA in Subjects with } \\
\text { Macular Edema Associated with Non-infectious } \\
\text { Uveitis (PEACHTREE):29 } \\
\text { ClinicalTrials.gov Identifier: NCT02595398 }\end{array}$ & Phase III & $\begin{array}{l}\text { ME due } \\
\text { to NIU }\end{array}$ & 160 & $\begin{array}{l}\text { Significant improvement in } \\
\text { vision and CST compared with } \\
\text { sham }\end{array}$ & $\begin{array}{l}\text { No serious AES related to treatment; } \\
\text { comparable rates of elevated IOP and } \\
\text { cataract between CLS-TA and sham groups }\end{array}$ \\
\hline $\begin{array}{l}\text { Suprachoroidal Injection of CLS-TA Alone or with } \\
\text { Aflibercept in Subjects with Diabetic Macular Edema } \\
\left(\text { HULK); }{ }^{34} \text { ClinicalTrials.gov Identifier: NCT02949024 }\right.\end{array}$ & Phase I/II & Diabetic ME & 20 & $\begin{array}{l}\text { CLS-TA alone or in combination } \\
\text { with intravitreal aflibercept, } \\
\text { improved VA in } \\
\text { treatment-naive patients }\end{array}$ & $\begin{array}{l}\text { Equal tolerability in both groups, no } \\
\text { significant cataract or elevation in IOP }\end{array}$ \\
\hline $\begin{array}{l}\text { Suprachoroidal Injection of Triamcinolone Acetonide } \\
\text { with IVT Aflibercept in Subjects with Macular Edema } \\
\text { Following RVO (SAPPHIRE); }{ }^{6,37} \\
\text { ClinicalTrials.gov Identifier: NCT02980874 }\end{array}$ & Phase III & $\begin{array}{l}\text { Retinal vein } \\
\text { occlusion } \\
\text { with ME }\end{array}$ & 460 & $\begin{array}{l}\text { Aflibercept alone or with } \\
\text { CLS-TA; comparable efficacy } \\
\text { across both groups at } 8 \text { weeks }\end{array}$ & N/A \\
\hline
\end{tabular}

$A E=$ adverse event; $C L S-T A=$ triamcinolone acetonide; $C S T=$ central subfield thickness; IOP = intraocular pressure; $M E=$ macular edema; $N I U=$ noninfectious uveitis; $V A=$ visual acuity.

Access to the suprachoroidal space has traditionally been via sclerotomy or ab interno surgical approaches (such as implantation of glaucoma drainage devices). ${ }^{20}$ The suprachoroidal space proved to be difficult to reliably, safely, and efficiently access, and, as such, intravitreal administration remains the prevailing drug delivery method. Recently, microneedles have been developed and evaluated for drug administration by ophthalmologists in an outpatient clinic setting. ${ }^{21}$ Once administered, the fluid delivered into the suprachoroidal space distributes circumferentially around the eye, allowing medication to slowly diffuse to the retinal pigment epithelium and retina. Higher levels of molecules are found within chorioretinal tissues with suprachoroidal space injections, along with significantly faster clearance when compared with intravitreal injections in drug distribution studies in animal models. ${ }^{22}$

Initial studies of triamcinolone acetonide, administered in the suprachoroidal space in rabbits, showed relatively minimal amounts of drug in the lens and anterior segment, but roughly 12-fold higher amounts of drug within the retina and choroid. ${ }^{23-25}$ These pharmacokinetic studies support the principle that localized administration leads to more targeted medication delivery while sparing non-diseased tissues. Further studies added evidence that high levels of medication were maintained in these areas of interest for up to 3 months, supporting the therapeutic potential for human disease. ${ }^{26}$

\section{Suprachoroidal injection of triamcinolone acetonide in clinical studies}

Suprachoroidal injection of triamcinolone acetonide (CLS-TA; XIPERETM [Clearside Biomedical, Alpharetta, GA]), a proprietary, preservative-free triamcinolone acetonide formulation has been well studied, with major studies shown in Table 1. It was first administered to human patients in a phase I/II trial, where nine patients with bilateral noninfectious uveitis and macular edema received an injection of CLS-TA in one eye. A single injection of $4 \mathrm{mg}$ triamcinolone acetonide in $100 \mu \mathrm{L}$ was administered on day 1 and observed for 26 weeks. The medication was well tolerated with no systemic adverse events. Mild ocular adverse events were observed, with the most common complaint being eye pain. Eight subjects showed improvements in best corrected visual acuity (BCVA) and seven patients, who entered with macular edema, showed at least a $60 \mu \mathrm{m}$ reduction in central subfield thickness (CST) on optical coherence tomography (OCT). ${ }^{27}$

The subsequent phase ॥ DOGWOOD study (ClinicalTrials.gov Identifier: NCT02255032) was a dose-randomized, controlled, masked trial observing the safety and efficacy of a single suprachoroidal injection of CLS-TA (4.0 and $0.8 \mathrm{mg}$ in a 4:1 ratio). Twenty-two individuals with macular edema caused by noninfectious uveitis were enrolled. The primary efficacy endpoint was change in CST on OCT from baseline. Patients were observed at 1 and 2 months after injection. The primary endpoint was met in the $4.0 \mathrm{mg}$ group with a reduction in CST of $>20 \%$ at month 2, with improvement of $>5$ Early Treatment of Diabetic Retinopathy Study (ETDRS) letters in 65\% of patients with a mean improvement of 9.2 letters. The safety endpoint was met, as no patients showed significant increases in IOP. During this short-term study, no significant development of cataracts was observed. ${ }^{28}$

More recently, the results of the phase III PEACHTREE sham-controlled, multicenter, randomized clinical trial (ClinicalTrials.gov Identifier: NCT02595398) were published in Ophthalmology. ${ }^{29}$ In this study, 160 patients with macular edema associated with a spectrum of noninfectious uveitis involving all anatomic locations were randomized 3:2 to receive a 
suprachoroidal injection of CLS-TA or a sham treatment at day 0 and a repeat injection at week 12 . Patients were monitored for 24 weeks with the primary endpoint being improvement of $\geq 15$ ETDRS letters from baseline. Secondary endpoints included reduction of CST from baseline OCT. Additional endpoints included changes in BCVA in ETDRS letters by visit, mean change in CST by visit, and changes in signs of intraocular inflammation by visit. ${ }^{5}$ Rescue therapy was administered if indicated by the study investigators.

The primary efficacy endpoint of $a \geq 3$-line BCVA gain (>15 ETDRS letters) was achieved at 24 weeks with $47 \%$ of the 96 individuals who received suprachoroidal CLS-TA. By comparison, only $16 \%$ of the 68 controls achieved this endpoint $(p<0.001)$. Improvement in BCVA of nearly 10 letters was observed by the week 4 time point, with continued improvement during follow up. A reduction in macular edema, as measured by the change in CST on OCT, was also significantly greater in the treatment group when compared to patients in the sham arm. Specifically, the mean reduction of CST was 153 microns in the CLS-TA arm compared with 18 microns in the sham arm $(p<0.001)$.

Adverse effects were also monitored closely during the phase III trial, with close attention to IOP and cataract. Elevated IOP and cataract development was comparable between groups (elevated IOP in $12 \%$ of treatment versus 16\% of sham groups; cataract progression in $7 \%$ of treatment versus $6 \%$ in the sham groups). One patient in the treatment arm developed a retinal detachment approximately 8 weeks after injection, but this was deemed unrelated to the procedure given the delayed onset and location. Notably, no cases of endophthalmitis, choroidal detachment or hemorrhage were observed.

The PEACHTREE study outcomes support triamcinolone acetonide as a potential therapeutic for macular edema associated with noninfectious uveitis. The visual outcomes highlighted above (mean gain of 14 ETDRS letters in 6 months) compare favorably with the previously reported literature on the visual outcomes of three currently administered local corticosteroids, which was reported in the Periocular and Intravitreal Corticosteroids for Uveitic Macular Edema (POINT) Trial. In the POINT trial (ClinicalTrials.gov Identifier: NCT02374060), approximately nine ETDRS letters of improvement was achieved by the intravitreal triamcinolone acetonide and intravitreal dexamethasone arms, and four letters were gained with periocular steroid administration. ${ }^{30}$

The safety data regarding IOP elevation requiring additional treatment also are compelling in PEACHTREE. ${ }^{29}$ IOP elevations occurred in approximately $11.5 \%$ in the treatment arm. It is notable that only $7.3 \%$ of patients in the treatment arm of PEACHTREE required medication to lower IOP. While PEACHTREE did not directly compare CLS-TA with other intravitreal corticosteroids, the POINT study provides helpful information regarding the incidence of IOP elevation with intravitreal corticosteroid. Specifically, in the POINT study, approximately $32-43 \%$ of patients required initiation of an IOP-lowering medication. ${ }^{30}$ No patients underwent IOP-lowering surgery or cataract surgery in either the PEACHTREE or POINT trials.

\section{Future applications}

Access to the suprachoroidal space provides another target for therapeutic development aimed at other sight-threatening diseases. Recent studies have examined the feasibility, efficacy, and safety in drug delivery within the suprachoroidal space for macular edema due to diabetes and retinal vein occlusions (RVO). Further studies have started to explore suprachoroidal space utility in neovascular-age-related macular degeneration as well as potential gene therapy for inherited retinal conditions. ${ }^{31-33}$

The phase I/II HULK trial (ClinicalTrials.gov Identifier: NCT02949024) examined the safety and efficacy of suprachoroidal CLS-TA alone or in combination with intravitreal aflibercept for diabetic macular edema. Twenty patients were separated into treatment-naive groups and those previously treated with either anti-VEGF agents or intraocular steroids. The treatment-naive arm received aflibercept (Eylea ${ }^{\oplus}$; Regeneron, Tarrytown, NY, USA) at initial baseline visit, and all participants received CLS-TA at the baseline visit, and then as needed based on CST measurements at months 2 through 5. Eighty-nine percent of all patients achieved $\mathrm{a} \geq 50 \%$ reduction in CST and a mean improvement in visual acuity of 5.2 letters (8.5 mean letter gain in the treatment-naive group versus a 1.1 mean letter gain in the previously treated group). ${ }^{34}$

The subsequent phase ॥ TYBEE trial (ClinicalTrials.gov Identifier: NCT03126786) described the treatment of 71 patients with diabetic macular edema who were randomized to aflibercept alone or aflibercept in combination with suprachoroidal triamcinolone acetonide. Both arms showed visual acuity gain; however, patients in the combination arm required fewer intravitreal aflibercept injections when compared to the aflibercept monotherapy arm in the as-needed treatment stage. ${ }^{35}$

Suprachoroidal CLS-TA was also evaluated for the treatment of macular edema associated with retinal vein occlusions. The recent phase III clinical trial, SAPPHIRE (ClinicalTrials.gov Identifier: NCT02980874), enrolled 460 patients with treatment-naive RVO and randomized them to receive aflibercept or aflibercept plus suprachoroidal CLS-TA. The primary endpoint of improved BCVA in the combined aflibercept plus suprachoroidal CLSTA treatment group compared to aflibercept alone was not observed. Specifically, approximately $50 \%$ of patients in both arms demonstrated $\geq 15$ ETDRS letters of improvement in BCVA at 8 weeks, showing comparable efficacy for patients in the triamcinolone acetonide plus intravitreal aflibercept combination and aflibercept only arms. ${ }^{36,37}$ Thus, in treatmentnaive patients with RVO, triamcinolone acetonide does not provide additional benefit when combined with aflibercept, compared to aflibercept alone. However, while no additional studies are currently planned, the benefit of suprachoroidal triamcinolone in combination with aflibercept may warrant future study, especially in recalcitrant RVOs, given the potential for improved durability with medication delivery to the suprachoroidal space.

Besides instillation of triamcinolone into the suprachoroidal space, other ocular therapeutics may benefit from suprachoroidal delivery. Suprachoroidal delivery of gene therapy via an AAV8 vector has been reported to achieve similar expression of an anti-VEGF Fab (monoclonal antibody fragment) as achieved with subretinal delivery of the AAV8 vector with corresponding functional suppression of vascular leakage in a rat model. ${ }^{33}$ Suprachoroidal delivery of axitinib, a tyrosine kinase inhibitor with anti-VEGF properties, ${ }^{31}$ as well as viral-like particle conjugates delivered to the suprachoroidal space for choroidal melanoma, are also currently under investigation. ${ }^{38}$

\section{Implementation in clinical practice}

The studies summarized in this review highlight the suprachoroidal injection drug delivery platform and potential niche of CLS-TA in the therapeutic armamentarium for retinal and inflammatory diseases. The PEACHTREE trial showed promising visual and anatomical results in patients with uveitic 
macular edema. It also demonstrated a low risk of IOP elevation and minimal risk of cataract development with a favorable safety profile compared with sham. These findings compare favorably with the reported literature related to intraocular and periocular administration of corticosteroid for macular edema associated with uveitis.
Basic and translational research is ongoing for additional disease indications that will further leverage the suprachoroidal drug delivery technology. Additional studies may provide ophthalmologists a novel therapeutic approach for posterior segment disease, including uveitis and retinal vascular disease conditions in the future. $\square$
1. Rothova A, Suttorp-van Schulten MS, Frits Treffers W, Kijlstra A Causes and frequency of blindness in patients with intraocular inflammatory disease. Br J Ophthalmol. 1996:80:332-6.

2. Nussenblatt RB. The natural history of uveitis. Int Ophthalmol. 1990;14:303-8.

3. Darrell RW, Wagener HP, Kurland LT. Epidemiology of uveitis. Incidence and prevalence in a small urban community. Arch Ophthalmol. 1962;68:502-14.

4. Krumpaszky HG, Ludtke R, Mickler A, et al. Blindness incidence in Germany. A population-based study from WurttembergHohenzollern. Ophthalmologica. 1999:213:176-82.

5. Jabs DA, Nussenblatt RB, Rosenbaum JT. Standardization of Uveitis Nomenclature (SUN) Working Group. Standardization of uveitis nomenclature for reporting clinical data. Results of the First International Workshop. Am J Ophthalmol. 2005;140:509-16.

6. Massa H, Pipis SY, Adewoyin T, et al. Macular edema associated with non-infectious uveitis: pathophysiology, etiology, prevalence, impact and management challenges Clin Ophthalmol. 2019:13:1761-77.

7. Lee $\mathrm{JH}, \mathrm{Mi} \mathrm{H}$, Lim R, et al. Ocular autoimmune systemic inflammatory infectious study-report 3: posterior and panuveitis. Ocul Immunol Inflamm. 2019;27:89-98.

8. Kok H, Lau C, Maycock N, et al. Outcome of intravitrea triamcinolone in uveitis. Ophthalmology. 2005;112:1916.e1-7.

9. Venkatesh P, Kumar CS, Abbas Z, Garg S. Comparison of the efficacy and safety of different methods of posterior subtenon injection. Ocul Immunol Inflamm. 2008:16:217-23.

10. Acharya NR, Hong KC, Lee SM. Ranibizumab for refractory uveitisrelated macular edema. Am J Ophthalmol. 2009;148:303-9.e2.

11. Rhee DJ, Peck RE Belmont J et al. Intraocular pressure alteration following intravitreal triamcinolone acetonide. Br J Ophthalmol. 2006;90:999-1003

12. Bollinger KE, Smith SD. Prevalence and management of elevated intraocular pressure after placement of an intravitreal sustainedrelease steroid implant. Curr Opin Ophthalmol. 2009:20:99-103.

13. Bernstein HN, Mills DW, Becker B. Steroid-induced elevation of intraocular pressure. Arch Ophthalmol. 1963;70:15-8.

14. Smithen LM, Ober MD, Maranan L, Spaide RF. Intravitreal triamcinolone acetonide and intraocular pressure. Am J Ophthalmol. 2004;138:740-3.

15. Wilson K, MCCartney MD, Miggans ST, Clark AF. Dexamethasone induced ultrastructural changes in cultured human trabecular meshwork cells. Curr Eye Res. 1993:12:783-93.
16. Behbehani AH, Owayed AF, Hijazi ZM, et al. Cataract and ocular hypertension in children on inhaled corticosteroid therapy. Pediatr Ophthalmol Strabismus. 2005:42:23-7.

17. Wang JJ, Rochtchina E, Tan AG, et al. Use of inhaled and oral corticosteroids and the long-term risk of cataract. Ophthalmology. 2009;116:652-7

18. Emi K, Pederson JE, Toris CB. Hydrostatic pressure of the suprachoroidal space. Invest Ophthalmol Vis Sci. 1989;30:233-8.

19. Tyagi P, Kadam RS, Kompella UB. Comparison of suprachoroida noninvasive fluorophotometry PLOS One . 2012:7:e48188.

20. Chiang B, Jung JH, Prausnitz MR. The suprachoroidal space as route of administration to the posterior segment of the eye. Adv Drug Deliv Rev. 2018;126:58-66.

21. Patel SR, Berevzosky DE, McCarey BE, et al. Targeted administration into the suprachoroidal space using a microneedle for drug delivery to the posterior segment of the eye. Invest Ophthalmol Vis Sci. 2012;53:4433-41.

22. Chiang B, Wang K, Ethier CR, Prausnitz MR. Clearance kinetics and clearance routes of molecules from the suprachoroidal space after microneedle injection. Invest Ophthalmol Vis SCi. 2017;58:545-54.

23. Rowe-Rendleman CL, Durazo SA, Kompella UB, et al. Drug and gene delivery to the back of the eye: from bench to bedside. Invest Ophthalmol Vis Sci. 2014;55:2714-30.

24. Kim YC, Edelhauser HF, Prausnitz MR. Particle-stabilized emulsion droplets for gravity-mediated targeting in the posterior segment of the eye. Adv Healthc Mater. 2014:3:1272-82.

25. Edelhauser HF, Verhoeven RS, Burke B, et al. Intraocular distribution and targeting of triamcinolone acetonide suspension administered into the suprachoroidal space. Invest Ophthalmo/ Vis SCi. 2014;55:5259.

26. Edelhauser H, Patel S, Meschter C, et al. Suprachoroidal microinjection delivers triamcinolone acetonide to therapeutically-relevant posterior ocular structures and limits exposure in the anterior segment Invest Ophthalmol Vis Sci. 2013:54:5063.

27. Goldstein DA, Do D, Noronha G, et al. Suprachoroida corticosteroid administration: a novel route for local treatment of noninfectious uveitis. Trans/ Vis Sci Technol. 2016;5:14

28. Yeh S, Kurup SK, Wang RC, et al. Suprachoroidal injection of triamcinolone acetonide, CLS-TA, formacular edema due noninfectious uveitis: a randomized, phase 2 study (DOGWOOD). Retina. 2019;39:1880-8.

29. Yeh S, Khurana RN, Shah M, et al. Efficacy and safety of suprachoroidal CLS-TA for macular edema secondary to noninfectious uveitis: phase 3 randomized trial. Ophthalmology. 2020;127:948-55

30. Thorne JE, Sugar EA, Holbrook JT, et al. Periocular triamcinolone vs. intravitreal triamcinolone vs. intravitreal dexamethasone implant for the treatment of uveitic macular edema: the PeriOcular vs. INTravitreal corticosteroids for uveitic macular edema (POINT) trial. Ophthalmology. 2019:126:283-95.

31. Kaiser PK, Ciulla T, Kansara V. Suprachoroidal CLS-AX (axitinib injectable suspension), as a potential long-acting therapy for neovascular age-related macular degeneration (nAMD). Invest Ophthalmol Vis Sci. 2020;61:3977.

32. Muya L, Kansara V, Ciulla T. Pharmacokinetics and ocular tolerability of suprachoroidal CLS-AX (axitinib injectable suspension) in rabbits. Invest Ophthalmol Vis Sci. 2020;61:4925.

33. Ding K, Shen J, Hafiz Z, et al. AAV8-vectored suprachoroidal gene transfer produces widespread ocular transgene expression. J Clin Invest. 2019:129:4901-11.

34. Wykoff CC, Khurana RN, Lampen SIR, et al. Suprachoroida triamcinolone acetonide for diabetic macular edema: the HULK trial. Ophthalmol Retina. 2018;2:874-7.

35. US National Library of Medicine. Suprachoroidal CLS-TA with intravitreal aflibercept versus aflibercept alone in subject with diabetic macular edema (TYBEE), 2019. Available at: https://clinicaltrials.gov/ ct2/show/NCT03126786 (accessed November 11, 2020)

36. US National Library of Medicine. Suprachoroidal injection of triamcinolone acetonide with IVT aflibercept in subjects with macular edema following RVO (SAPPHIRE), 2019. Available at: https://clinicaltrials.gov/ct2/show/NCT02980874 (accessed November 11, 2020)

37. Clearside Biomedical. Clearside Biomedical announces SAPPHIRE phase 3 study of combination therapy in retinal vein occlusion did not meet its primary endpoint, 2018. Available at: https:// ir.clearsidebio.com/news-releases/news-release-details/ clearside-biomedical-announces-sapphire-phase-3-study (accessed November 11, 2020)

38. Savinainen A, Grossniklaus H, Kang S, et al. Ocular distribution and efficacy after suprachoroidal injection of AU-011 for treatment of ocular melanoma. Invest Ophthalmol Vis SCi. 2020;61:3615. 